\title{
Microbiota and compartment matter in the COVID-19 response
}

\author{
During acute COVID-19, there is little correlation between the nose and blood in terms of antibodies or cytokines; \\ instead, these factors are associated with nasal microbiota.
}

\section{Simon P. Jochems, Daniela M. Ferreira and Hermelijn H. Smits}

1 yperinflammatory responses during infection with the coronavirus SARS-CoV-2 promote severe disease progression by triggering coagulation and immunopathological damage during COVID-19. At the same time, innate and SARS-CoV-2-specific adaptive immune responses are essential for clearance of the virus and protection against (re-) infection. Although SARS-CoV-2 infects predominantly the respiratory tract, the vast majority of studies thus far have investigated immune responses in blood. In the current issue of Nature Immunology, Smith et al. use integrated analyses to characterize cytokines and antibodies in paired nasopharyngeal swab material and peripheral blood in a cross-sectional cohort of hospitalized patients at 8-12 days after symptom onset ${ }^{1}$. They associated these immunological parameters with disease severity, nasal and systemic SARS-CoV-2 viral load, and the nasal microbiome.

Confirming previous reports ${ }^{2}$, they found that titers and neutralization capacity of SARS-CoV-2-specific immunoglobulin G (IgG) and $\operatorname{IgA}$ progressively increased with disease severity, both in the blood and in the nasopharynx. However, even though antibody titers increased with severity in both blood and nose, on an individual level there was no correlation between blood and the nasopharynx in their antibody levels or neutralizing capacity. Strikingly, 80-90\% of patients had SARS-CoV-2-specific antibodies in serum ('seroconversion'), whereas the presence of nasal SARS-CoV2-specific antibodies ('nasoconversion') was less common, with only $30 \%$ of patients having this in the group with moderate disease. This nasoconversion rate increased to $70 \%$ in critically ill patients. Although antibody dynamics were not assessed in this cross-sectional cohort, others have reported that patients with mild disease have a delayed seroconversion, starting from 10-12 days after symptom onset, relative to that of patients with severe disease ${ }^{2}$. It

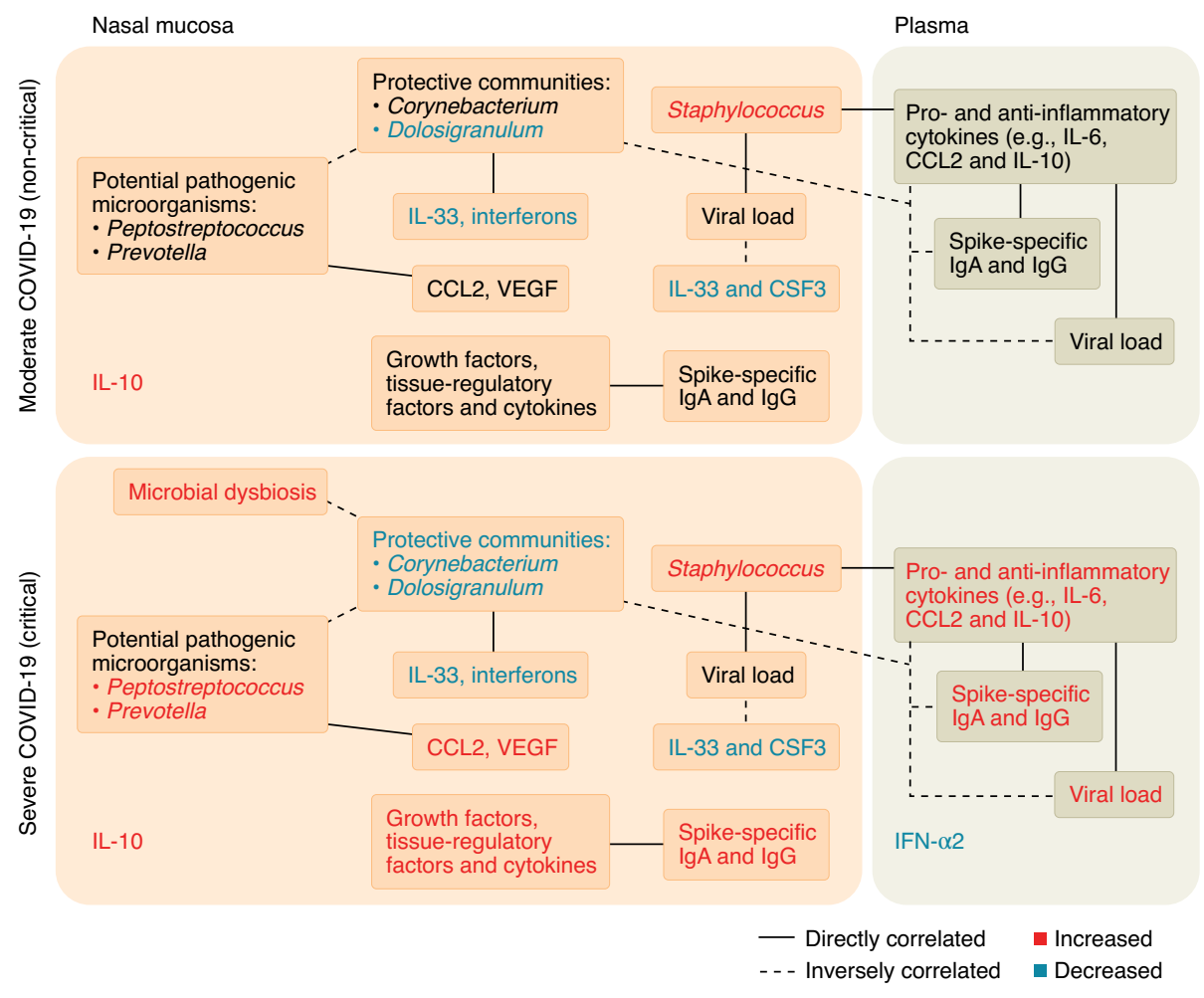

Fig. 1 | Changes in inflammation, antibodies and microbiota during COVID-19. Changes in the nasal compartment (left) and systemic compartment (right) are shown separately, while patients are also stratified as having moderate disease (top) or severe or critical disease (bottom). Font color indicates whether a parameter is increased (red) or decreased (green). Associated features are connected by solid lines (positive correlation) and dashed lines (negative correlation). Of note, not all identified significant parameters and links are shown to reduce complexity.

is thus possible that more of the patients with moderate disease would undergo nasoconversion later, but studies that include longitudinal sampling are needed to address this. The lower nasoconversion rate in the group with moderate disease than that of critically ill patients is somewhat surprising, as mild infection has been reported to elicit mucosal SARS-CoV-2-specific IgA ${ }^{2}$, which highlights the complexity of balancing immunity with tissue regulation.
Investigating cytokine responses, Smith et al. found several cytokines and immune system-relevant proteins that were increased in plasma of patients (VEGF, FGF, IL-1RA, IL-6, TNF, IL-10, CCL2, CXCL10, CCL3, CCL19, PD-L1, G-CSF and granzyme B), most of which showed an increasing concentration in plasma with increasing disease severity ${ }^{1}$. The antiviral cytokine IFN- $\alpha 2$ was present at lower levels in plasma of critically ill patients, as previously 
reported by this same group and others ${ }^{3}$. Of all the cytokines altered in blood, only the anti-inflammatory cytokine IL-10 and the monocyte-recruiting factor CCL2 were also increased in the nose. In contrast, nasal levels of IL-33, and of the interferons IFN $-\alpha 2$, IFN- $\beta$, IFN- $\gamma$ and IFN- $\lambda 3$, were lower in patients than in healthy control donors. Thus, similar to results obtained for antibodies, there was a lack of correlation between cytokines in the nose and those in the circulation of patients, indicative of the compartmentalization of immune responses.

Even though various viral proteins from SARS-CoV-2 can affect the expression of type I interferons in airway epithelial cells in vitro ${ }^{4}$, and interferons themselves might block viral replication, nasal interferon levels did not significantly correlate inversely with viral load. Nonetheless, this paper by Smith et al. ${ }^{1}$, as well as another recent study that found increased expression of interferon-stimulated genes in the nasal epithelium of patients with mild or moderate COVID-19, but not in that of patients with severe COVID- $19^{5}$, indicate the importance of local interferon responses in mucosa. Again, longitudinal analyses are needed to establish a critical role for these cytokines in reducing viral load and preventing severe disease progression. Moreover, it will be important to consider the local protective nasal cellular responses as well in future studies, especially given the limited correlation between nose and blood. Combining the antibody and cytokine data revealed that patients who had undergone nasoconversion had more nasal inflammation than that of patients who did not. This could point to the importance of mucosal inflammation for the development of local antibodies, as observed previously for vaccination with a live attenuated influenza vaccine ${ }^{6}$. Conversely, this correlation could point to the triggering of inflammation by antibodies. Indeed, high levels of IgG antibodies with low levels of fucosylation in patients with severe disease have been shown to be able to induce inflammation by human macrophages via the Fcy receptor ${ }^{7}$.

The nasal viral load, unlike the plasma viral load, did not correlate with disease severity, as has been shown previously. Nasal viral load was also not associated with concentrations of local cytokines and antibodies, with the exception of an inverse correlation with IL-33 and CSF3. This indicates that host responses to the virus, rather than viral load itself, are driving these inter-related processes of inflammation, pathology and immunity. The loss of IL-33, which is important for type 2 immunity and tissue remodeling, in the nasal mucosa with increasing disease severity is somewhat surprising, as IL-33 is increased in other viral infections and in inflammation in the airways. After stratification by disease severity, but not by viral load, patients with critical COVID-19 had significantly higher nasal levels of not only the cytokines CXCL1 and IL-7 but also the growth factors FLT3-1, EGF, VEGF, PDGF-AA and TGF- $\alpha$. This suggests the induction of regulatory and/or repair responses in response to tissue damage, and it is thus likely that local repair responses other than those induced via IL-33 are dominant during severe COVID-19.

To find what was driving the local and systemic immune responses, Smith et al. then turned to the nasal microbiota, which they analyzed by $16 \mathrm{~S}$ sequencing ${ }^{1}$. Patients with severe and critical disease showed a diminished microbial diversity, as well as lower abundance of the beneficial commensals Corynebacterium and Dolosigranulum, relative to that of the healthy donors and patients with moderate disease. The reduction in microbiota diversity and beneficial commensals was associated with expansion of not only, in particular, Staphylococcus, but also Prevotella and Peptostreptococcus. Although only a few reports have assessed potential interactions between bacterial colonization of the upper airway and infection with SARS-CoV-2, this mirrors earlier reports of decreased microbiota diversity and Corynebacterium levels with a concurrent expansion of opportunistic pathogens (Haemophilus, Stenotrophomonas, Acineobacter, Moraxella and Pseudomonas) in patients with COVID-198. Through the integration of nasal bacteria with mucosal and plasma cytokines, a clearer picture starts to emerge for the complex interactions among host, virus and commensals that are associated with disease severity (Fig. 1). For example, Smith et al. demonstrated that bacterial diversity was strongly associated with mucosal levels of IL-33, IFN- $\lambda 3$ and IFN- $\gamma$ in patients with COVID-191. Previously, it had been shown that the microbiota can modulate continuous expression of interferons by plasmacytoid dendritic cells, which suggests that a beneficial microbiota could help prevent severe disease ${ }^{9}$. At the same time, the abundance of bacterial pathobionts such as Staphylococcus in the nasal mucosa (unlike almost all of the local immunological parameters assessed) was strongly associated with systemic inflammation, such as IL-6. Plasma viral load also clustered with these parameters. It was previously shown that in the serum of patients with severe COVID-
19, microbial products can be found that correlate with inflammatory markers ${ }^{10}$. Given the increased abundance of tissue growth factors that Smith et al. observed in the nasal mucosa of critically ill patients ${ }^{1}$, the hypothesis emerges that substantial tissue damage in the respiratory tract of these people leads to 'leaking' of virus and potential pathogenic bacteria or bacterial products, which results in systemic hyperinflammation.

The interactions among host responses, nasal microbiota and infections with respiratory viruses, such as SARS-CoV-2, is of great scientific and clinical interest. In one pre-print, colonization by Streptococcus pneumoniae in people who experienced asymptomatic or mild infection with SARS-CoV-2 was associated with reduced inflammation, impaired levels of mucosal IgA and lower memory B cell and T cell responses to SARS-CoV-2 antigens ${ }^{11}$. Furthermore, a recent observational study reported that 13 -valent pneumococcal conjugate vaccine in older adults was associated with a reduction of approximately $30 \%$ in COVID-19 disease, hospitalization and death ${ }^{12}$. Apart from SARS-CoV-2, susceptibility and immune responses to other respiratory viral infections are also known to be modulated by the nasal microbiota. During experimental infection with human rhinovirus, baseline microbiota clusters are associated with subsequent host inflammatory responses, viral load and symptom severity ${ }^{13}$. In hospitalized children infected with respiratory syncytial virus, viral infection is associated with increased presence of pathobionts such as Streptococcus and Haemophilus influenzae. Moreover, increased proinflammatory gene signatures are present in the blood of children co-infected with both respiratory syncytial virus and these pathobionts ${ }^{14}$. The nasal microbiota was also shown to be associated with susceptibility to infection with influenza virus in household transmission studies in which Alloprevotella and Prevotella were linked to infection ${ }^{15}$. Also, similar to its potential effects on host responses to SARS-CoV-2 ${ }^{11}$, colonization by Streptococcus pneumoniae blunted nasal inflammation and impaired the development of mucosal IgA responses to influenza virus antigens in a controlled human co-infection study ${ }^{6}$.

Collectively, the manuscript by Smith et al. ${ }^{1}$ provides important new insights into the pathogenesis of COVID-19. Moreover, it is now also clear that viral load, antibodies, cytokines and immune proteins are regulated differentially in the nose versus the blood, with little correlation between the two compartments. This highlights the 
urgent need for further studies into the mucosal immune responses and microbiota during infection with SARS-CoV-2 for better understanding of the disease progression and local mechanisms that are important for either swift clearance of infection with only mild symptoms or the prevention of tissue damage.

Simon P. Jochems (DD $1 \bowtie$,

Daniela M. Ferreira (D) ${ }^{2}$ and

Hermelijn H. Smits (D)

${ }^{1}$ Department of Parasitology, Leiden University

Medical Center, Leiden, the Netherlands.
${ }^{2}$ Department of Clinical Sciences, Liverpool School of

Tropical Medicine, Liverpool, UK.

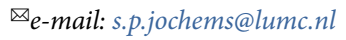

Published online: 21 October 2021

https://doi.org/10.1038/s41590-021-01041-w

$\neg$ References

1. Smith, N. et al. Nat. Immunol. https://doi.org/10.1038/s41590021-01028-7 (2021).

2. Cervia, C. et al. J. Allergy Clin. Immunol. 147, 545-557.e549 (2021).

3. Hadjadj, J. et al. Science 369, 718-724 (2020).

4. Lei, X. et al. Nat. Commun. 11, 3810 (2020).

5. Ziegler, C. G. K. et al. Cell 184, 4713-4733.e22 (2021).

6. Carniel, B. F. et al. JCI Insight 6, el41088 (2021).
7. Hoepel, W. et al. Sci. Transl. Med. https://doi.org/10.1126/ scitranslmed.abf8654 (2021).

8. Gupta, A. et al. Microbes Infect. https://doi.org/10.1016/j.micinf. 2021.104880 (2021).

9. Schaupp, L. et al. Cell 181, 1080-1096.e19 (2020).

10. Arunachalam, P. S. et al. Science 369, 1210-1220 (2020).

11. Mitsi, E. et al. Preprint at medRxiv https://doi.org/10.1101/ 2021.07.22.21260837 (2021).

12. Lewnard, J.A. et al. J. Infect. Dis. https://doi.org/10.1093/infdis/ jiab128 (2021).

13. Lehtinen, M. J. et al. Sci. Rep. 8, 11411 (2018).

14. de Steenhuijsen Piters, W. A. et al. Am. J. Respir. Crit. Care Med. 194, 1104-1115 (2016).

15. Lee, K. H. et al. PLoS One 14, e0207898 (2019).

Competing interests

The authors declare no competing interests.

\section{Is VITT really a HIT}

Thrombosis complicates SARS-CoV-2 infection and vaccination. Recent data are being used to identify the autoimmune antibody repertoires responsible for the excessive activation of coagulation and platelets.

\section{Zaverio M. Ruggeri and Wolfram Ruf}

$\mathrm{n}$ a recent Nature article, Huynh et al. ${ }^{1}$ identify the epitope on platelet factor 4 (PF4; also known as CXCL4) recognized by five antibodies isolated from people who developed thrombocytopenia and thrombosis after receiving the ChAdOx1 nCoV-19 vaccine against COVID-19 (commonly referred to as the 'University of Oxford-AstraZeneca' vaccine) $)^{2,3}$. This syndrome, which is characterized by thrombosis in unusual sites (notably, sinus veins of the brain), has an incidence of one case or less per 100,000 vaccinations, a female/male ratio of $\sim 2: 1$ and a mortality of about three in ten cases. Greinacher et al. concluded that platelet-activating anti-PF4 antibodies, akin to heparin-induced thrombocytopenia (HIT), cause this new syndrome, for which they propose the name 'vaccine-induced immune thrombotic thrombocytopenia' (VITT) ${ }^{3}$. As none of the patients with VITT had received heparin during the time required for the appearance of symptoms, the authors suggested that VITT resembles the previously described autoimmune HIT, in which thrombotic thrombocytopenia is heparin independent ${ }^{4}$.

HIT is phenotypically diverse, but always involves IgG antibodies that react with PF4, a positively charged tetrameric protein (polycationic) of the CXC chemokine family stored in platelet $\alpha$-granules and released in response to platelet activation. Typical antibodies involved in HIT react with PF4 bound to negatively charged heparin polymers (polyanionic) that form ultra-large pathogenic immunocomplexes and activate platelets through the receptor Fc $\gamma$ RIIA $(\mathrm{CD} 32 \mathrm{a})^{5}$. By contrast, HIT anti-PF4 autoimmune antibodies directly cluster PF4 tetramers and thereby activate platelets without heparin. Thus, a multiplicity of factors that affect size and stability of anti-PF4 immunocomplexes can explain the pathogenesis and phenotypic diversity of HIT. Of particular importance is the binding affinity of anti-PF4 antibodies, but equally relevant are the electrostatic interactions of polycationic PF4 with polyanions that, in addition to exogenous heparin, might include glycosaminoglycans and polyphosphates associated with platelets or other vascular and tissue cells. Therefore, organ-specific characteristics and individual platelet phenotypes can affect the assembly, size and physicochemical properties of anti-PF4-PF4 antigenantibody complexes. Then, engagement of Fc $\gamma$ RIIA leads to enhanced procoagulant activity with increased thrombin generation, fibrin deposition and thrombin feedback regulation of platelet activation, which creates a self-perpetuating highly prothrombotic environment in the vessel lumen and on cell surfaces (Fig. 1). The end result is the syndrome of thrombocytopenia caused by platelet consumption and immunothrombosis, in which anti-PF4 IgG antibodies are common to HIT and VITT.

The study by Huynh et al provides new insights into pathogenic anti-PF4 antibodies by identifying the epitope specificity of five VITT antibodies and ten HIT antibodies, with four of the latter being heparin dependent $^{1}$. The results of testing 70 PF4 mutants, each with a single residue replaced with alanine, establish that the epitope common to all VITT antibodies, but in none of the HIT antibodies, includes four residues shared with the heparin-binding site of PF4. VITT and HIT anti-PF4 antibodies, whether heparin dependent or independent, have similar binding characteristics, which suggests a comparable ability to form platelet-activating immunocomplexes. These data indicate that structural characteristics of anti-PF4 antibody epitopes might explain pathogenicity, which delineates a method for characterizing antibodies linked to well-defined disease phenotypes.

The origin and development of VITT autoantibodies in the context of immunization remains an open question, although much of what is known about the origin of HIT antibodies might apply to VITT ${ }^{4,6}$. The delayed onset of the syndrome would indicate that VITT anti-PF4 antibodies undergo expansion after the initial vaccination-associated 